\title{
MODO DE ENSACAMENTO DE TOMATE COM TECIDO-NÃO-TECIDO (TNT) NO MANEJO DA BROCA-PEQUENA-DO-FRUTO
}

\author{
FRAGOSO, Débora Ferreira Melo ${ }^{1}$ \\ GRECCO, Eduardo Domingos ${ }^{1}$ \\ TÚLER, Amanda Carlos ${ }^{2}$ \\ CARVALHO, José Romário de ${ }^{1}$ \\ ARAUJO JUNIOR, Luis Moreira de ${ }^{1}$ \\ DAMASCENA, Alixelhe Pacheco ${ }^{* 1}$ \\ BUENO, Regiane Cristina Oliveira de Freitas ${ }^{3}$ \\ PRATISSOLI, Dirceu ${ }^{1}$
}

\begin{abstract}
RESUMO: O ensacamento de tomates, Solanum lycopersicum (Linnaeus, 1753) (Solanales: Solanaceae), com tecido-não-tecido (TNT) tem sido uma técnica viável no manejo de Neoleucinodes elegantalis (Guenée, 1854) (Lepidoptera: Crambidae) em estabelecimentos agrícolas familiares. Logo, no intuito de propiciar agilidade no ato do ensacamento e facilitar a observação dos cachos, objetivou-se avaliar a qualidade de tomates em cachos ensacados com fundo aberto em comparação ao fundo fechado e aos cachos sem ensacamento no manejo de N. elegantalis. A qualidade dos frutos foram analisados pelas variáveis: número de orifícios de saída de N. elegantalis por cacho; peso, $\mathrm{pH}$ e teor de sólidos solúveis totais $\left(\mathrm{Brix}^{\circ}\right)$. Na redução do número de frutos brocados, o ensacamento com o fundo aberto foi mais eficiente apenas que os cachos sem ensacamento, sendo os valores 0,26000 orifícios/cacho e 0,79333 orifícios/cacho, respectivamente. Nas demais variáveis, não houve diferença significativa entre os tratamentos.
\end{abstract}

Palavras-Chave: Estabelecimento agrícola familiar, Frutos ensacados, Manejo fitossanitário de pragas; Neoleucinodes elegantalis.

\section{TOMATO PACKING MODE WITH NON-WOVEN FABRIC (NWF) IN THE MANAGEMENT OF THE SMALL FRUIT DRILL}

\begin{abstract}
SUMMARY: The bagging tomatoes, Solanum lycopersicum (Linnaeus, 1753) (Solanales: Solanaceae) with nonwoven fabric (NWF) has been a viable technique in the management of Neoleucinodes elegantalis (Guenée, 1854) (Lepidoptera: Crambidae) in familiar agricultural establishments. Thus, in order to provide agility in the act of bagging and facilitate the observation of the bunches, the objective was to evaluate the quality of tomato bunches covered by open bottom bags in comparison to the closed bottom and bunches without bagging in $N$. elegantalis management. The fruit quality was analyzed by the following variables: Number of $N$. elegantalis exit holes per bunch; weight, $\mathrm{pH}$ and total soluble solids content $\left(\mathrm{Brix}^{\circ}\right)$. In reducing the number of brocaded fruits, bagging with open bottom was more efficient only than bunches without bagging, being 0.26000 holes/bunch and 0.79333 holes/bunch, respectively. In the other variables, there was no significant difference between treatments.
\end{abstract}

Keywords: Family farm, Bagged fruits, Phytosanitary pest management; Neoleucinodes elegantalis.

\footnotetext{
${ }^{1}$ Núcleo em Desenvolvimento Científico e Tecnológico em Manejo Fitossanitário de Pragas (NUDEMAFI), Centro de Ciências Agrárias da Universidade Federal do Espírito Santo, Rua Alto Universitário, s/n, Caixa Postal 16, CEP: 29500-000, Alegre-ES.

${ }^{2}$ Universidade de São Paulo (USP), Campus Luiz de Queiroz. Escola Superior de Agricultura Luiz de Queiroz. São Dimas, 13.418-900, Piracicaba, SP, Brazil.

${ }^{3}$ Universidade Estadual Paulista (UNESP), Faculdade de Ciências Agronômicas, Departamento de Proteção de Plantas, 18610-034, Botucatu, São Paulo, Brazil.

*Corresponding author: Universidade Federal do Espírito Santo, Alto Universitário, s/nº, Guararema, Alegre, Espírito Santo, CEP 29500-000, Brasil. xellydamascena@ hotmail.com. (28)99948-5641.
} 


\section{INTRODUÇÃO}

O tomate, Solanum lycopersicum (Linnaeus, 1753) (Solanales: Solanaceae), é a hortaliça mais consumida in natura no mundo (HACHMANN et al., 2014). No ano de 2017, a produção mundial foi de 182.301,395 t (FAO, 2018). O maior produtor mundial de tomate com cerca de $32,64 \%$ de toda a produção foi a China, seguida pela Índia, Turquia, EUA, Egito, Iran, Itália, Espanha, México e o Brasil. No Brasil, a produção foi de 4,2 milhões de toneladas de tomate em 62,6 mil hectares de área plantada (IBGE, 2018). O consumo desta hortaliça ocorre em todos os estados do país, por todas as classes sociais e é cultivada em diferentes condições climáticas e estabelecimentos agrícolas (IBGE, 2018).

A cultura do tomate é afetada por diversas pragas, que diminuem a produtividade e/ou diminuem o valor das lavouras, comprometendo sua qualidade final. A broca-pequena-do-fruto, Neoleucinodes elegantalis (Guenée, 1854) (Lepidoptera: Crambidae), é uma das pragas mais importantes na produção de Solanaceae na América do Sul (MONTILA; SOLIS; KONDO, 2013). A fêmea de $N$. elegantalis deposita os ovos, no cálice ou no fruto jovem (com diâmetro de 0,5 a 4,0 cm) e após a eclosão, as larvas recém-nascidas passam alguns minutos na superfície do fruto antes de penetrá-la onde se alimentam até deixar o fruto para tornarem-se pupas (BLACKMER; EIRAS; SOUZA, 2001; MARCANO, 1991). Neste momento são formados orifícios de saída que inviabilizam a comercialização dos frutos, dada a formação de galerias em seu interior. Na América do Sul, a estimação do dano larval está entre 50 e $90 \%$ das perdas econômicas de plantações de tomate (MONTILA; SOLIS; KONDO, 2013; PICANÇO et al., 2007).

$\mathrm{Na}$ década de 60, o ensacamento de frutos era prática usual, principalmente, para o pessegueiro, pereira e ameixeira em estabelecimentos agrícolas familiares no Rio Grande do Sul (LIPP; SECCHI, 2002). A substituição pela aplicação de inseticidas ocorreu com a expansão do número de formulados de agrotóxicos no início dos anos 70. Entretanto, a mudança no perfil do consumidor tem exigido a produção de alimentos com níveis reduzidos ou mesmo isentos de agrotóxicos (WAMSER et al., 2008). Essa tendência indica a necessidade de avaliar novas estratégias de controle de pragas. Neste sentido, o retorno da utilização de ensacamento de frutos, tem se mostrado uma alternativa eficaz para o controle de pragas, principalmente em pequenas propriedades.

$\mathrm{O}$ uso da técnica de ensacamento em hortaliças requer a identificação de materiais adequados (duráveis e fáceis de aplicar) e economicamente viáveis. No caso do tomateiro, as sacolas de tecido-não-tecido (TNT) para o controle de N. elegantalis proporcionou incremento de $21,5 \%$ de produtividade em relação aos frutos não ensacados, e uma redução de $40,7 \%$ no custo médio estimado em relação ao controle químico (FILGUEIRAS et al., 2017). Neste caso, o custo 
do controle químico foi baseado no trabalho manual mais inseticidas, enquanto que o custo do sistema de ensacamento foi baseado no trabalho manual mais ensacamento com sacolas TNT. Logo, no intuito de propiciar agilidade no ato do ensacamento com sacolas de TNT e facilitar a observação dos cachos, objetivou-se avaliar a qualidade de tomates em cachos ensacados com fundo aberto em comparação ao fundo fechado e aos cachos sem ensacamento no manejo de $N$. elegantalis.

\section{MATERIAL E MÉTODO}

Os experimentos foram conduzidos em estabelecimento agrícola familiar nas safras 2012 e 2013, no município de Conceição do Castelo, ES situado a 637 metros de altitude e coordenadas geográficas Latitude: $20^{\circ} 21^{\prime} 50^{\prime \prime}$ Sul; Longitude: 41 14' 57" Oeste. A variedade de tomate utilizada foi a CLX. Os tratos culturais como transplante, tutoramento, amarrio, capação e desbrota foram realizados seguindo recomendações para a cultura (ABAURRE, 2010). O sistema de condução com duas hastes por planta em tutoramento vertical foi adotado, por ser o mais utilizado pelos pequenos produtores de tomate estaqueado no Estado do Espírito Santo. Este método baseou-se na condução das plantas por meio de estacas de bambu os quais as plantas foram amarradas a cada 7 dias. O espaçamento 1,3 x 0,7 m (linhas x plantas) foi utilizado.

O ensacamento foi efetuado nos três primeiros cachos por planta, a partir do momento que os primeiros frutos atingiram $20 \mathrm{~mm}$ de diâmetro para prevenir posturas. A cobertura dos cachos foi realizada com TNT $(30 \times 35 \mathrm{~cm})$, conhecido como tecido-não-tecido, classificado na indústria têxtil como um tecido técnico, devido à sua ampla aplicação na agricultura e indústria em geral. As sacolas de TNT foram preparados com o auxílio de uma prensa elétrica. Dois tipos de sacolas foram confeccionados; uma com fundo fechado, que protege totalmente o cacho (Controle Negativo), e outra com fundo aberto, que permitia a observação do cacho. Em campo, as sacolas foram fixadas no pedúnculo do cacho, acima dos frutos, utilizando fita crepe. $\mathrm{Na}$ área sem sacola (Controle Positivo), foi realizado o manejo de broqueadores conforme calendário préestabelecido, no qual a cada três dias, empregava-se o uso de inseticidas específicos e recomendados para esses insetos.

A qualidade dos frutos foi avaliada assim que os mesmos apresentavam-se em processo de maturação. As variáveis analisadas foram: Número de orifícios de saída de $N$. elegantalis por cacho, peso, $\mathrm{pH}$ e teor de sólidos solúveis totais $\left(\mathrm{Brix}^{\circ}\right)$. Os tomates foram pesados de acordo com cada tratamento. $\mathrm{O} \mathrm{pH}$ do fruto foi avaliado através da trituração dos frutos e posterior avaliação em pHmetro de bancada. $\mathrm{O}$ Brix ${ }^{\circ}$ foi determinado por meio da trituração dos frutos e posterior leitura em refratômetro (PEARSON, 1973). 
O experimento foi instalado em um delineamento experimental em blocos casualizado em um talhão com 150 plantas composto de 3 tratamentos (sacola com fundo x sacola sem fundo $\mathrm{x}$ sem sacola) e 10 blocos. Cada bloco foi composto por 15 plantas. Os dados foram submetidos ao teste de normalidade Shapiro-Wilk $(\mathrm{P}>0,05)$. Como não houve normalidade nos dados, os mesmos foram submetidos ao teste não paramétrico Kruskall-Wallis $(\mathrm{P}>0,05)$ pelo programa estatístico Assistat.

\section{RESULTADO E DISCUSSÃO}

Os tratamentos apresentaram diferença significativa $(H=129.8069, \mathrm{p}<0.05)$ na variável Número de orifícios de saída de $N$. elegantalis por cacho (Tabela 1). O tratamento com a sacola de TNT fechado propiciou o melhor resultado (0,00667 orifícios/cacho), que diferiu estatisticamente do ensacamento com o fundo aberto (0,26000 orifícios/cacho), e também dos cachos de tomate onde o ensacamento não foi realizado (0,79333 orifícios/cacho).

Tabela 1 - Número de orifícios de saída de Neoleucinodes elegantalis por cacho de tomate nos tratamentos.

\begin{tabular}{ccccc}
\hline Tratamento & \multicolumn{4}{c}{ Número orifícios/cacho $^{\mathbf{1}}$} \\
\hline Sacola com fundo fechado & 0,00667 & \pm & 0,00027 & $\mathrm{a}$ \\
Sacola com fundo aberto & 0,26000 & \pm & 0,00205 & $\mathrm{~b}$ \\
\hline Sem ensacamento & 0,79333 & \pm & 0,00279 & $\mathrm{c}$ \\
\hline
\end{tabular}
Wallis.

1 Médias seguidas por mesma letra não diferem si ao nível de $5 \%$ de probabilidade pelo teste Kruskal-

De acordo com os dados apresentados, notou-se que o ensacamento de TNT com o fundo fechado foi eficiente na proteção dos frutos, uma vez que nessa forma de ensacamento o mesmo apresentou uma redução expressiva de frutos atacados quando comparado aos demais tratamentos. A presença de orifícios em frutos nesse tipo de sacolas pode ser explicada devido, no momento da instalação do experimento, alguns frutos já estarem mais desenvolvidos, ultrapassando o diâmetro de $20 \mathrm{~mm}$, quando esse estão aptos a serem perfurados pela larva da broca pequena do tomateiro (PRATISSOLI, 2015). O presente trabalho mostrou que a técnica de ensacamento com sacolas de fundo aberto apresentou uma certa proteção aos frutos em relação ao tratamento que não houve ensacamento. Porém, o ensacamento com sacolas de fundo aberto foi menos eficiente que o ensacamento com sacolas de fundo fechado, visto que o fundo aberto propiciou a entrada de adultos que conseguiram ovipositar nos frutos. A preferência da mariposa $N$. elegantalis em depositar seus ovos em frutos pequenos localizados nos quatro primeiros frutos 
basais da penca de tomates já foram determinados por Blackmer, Eiras e Souza (2001). Porém, o presente trabalho mostrou, que além dessa preferência, a mariposa $N$. elegantalis também possui capacidade de procura por alimento. Logo, para que o ensacamento com sacola de TNT seja mais eficiente no manejo desta praga é necessário o fechamento das sacolas, embora demande mais tempo de trabalho.

As variáveis peso, $\mathrm{pH}$ e $\mathrm{Brix}^{\circ}$ por fruto de tomate não apresentaram diferença significativa entre os tratamentos (Tabela 2). Na média dos tratamentos, os valores do peso, $\mathrm{pH} \mathrm{e}$ Brix $^{\circ}$ foram $0,179 \mathrm{Kg}, 4,39$ e 4,47, respectivamente. No trabalho de Leite et al. (2014) também foram analisados as variáveis peso e Brix $^{\circ}$ por fruto de tomates, em que os valores do ensacamento não apresentaram diferença significativa da testemunha. $\mathrm{O}$ valor da variável $\mathrm{pH}$ no trabalho de Jordão e Nakano (2002) também não apresentou diferença significativa no ensacamento de tomate em relação a testemunha. Possivelmente, isto tenha ocorrido devido permeabilidade do TNT que permite a realização das trocas gasosas dos frutos (LEITE et al., 2014).

Tabela 2 - Avaliação da média do peso, valor do Brix $^{\circ}$ e $\mathrm{pH}$ por fruto de tomate nos tratamentos.

\begin{tabular}{cccc}
\hline Tratamento & Peso $(\mathbf{k g})^{\mathbf{1}}$ & $\mathbf{p H}^{\mathbf{1}}$ & Brix $^{\circ} \mathbf{1}$ \\
\hline $\begin{array}{c}\text { Sacola com fundo } \\
\text { fechado }\end{array}$ & 0,187 & 4,34 & 4,53 \\
$\begin{array}{c}\text { Sacola com fundo } \\
\text { aberto }\end{array}$ & 0,183 & 4,42 & 4,31 \\
Sem ensacamento & 0,168 & 4,42 & 4,58 \\
\hline Média & $\mathbf{0 , 1 7 9}$ & $\mathbf{4 , 3 9}$ & $\mathbf{4 , 4 7}$ \\
\hline
\end{tabular}

${ }^{1}$ Não significativo pelo teste de Dunn a 5\% de significância.

As pesquisas que visam a utilização da técnica de ensacamento de frutos, tem como ponto de partida o tipo de invólucro que será empregado. No entanto, o maior desafio é utilizar um material que seja resistente a ponto de proteger contra as pragas e que não exija alto custo com mão-de-obra para aplicação, além de não interferir no desenvolvimento dos frutos. Por meio desta e outras pesquisas, verificou-se que o TNT atende a estes requisitos. Todavia, apenas o presente trabalho mostrou que o simples fato de ensacar os cachos é suficiente para reduzir, significativamente, o número de frutos brocados. Porém, o ensacamento com fundo fechado potencializa a proteção dos frutos, embora demande mais tempo de trabalho. 


\section{CONCLUSÃO}

O ensacamento de cachos de tomate com sacola de TNT com o fundo fechado foi mais eficiente na redução do número de frutos brocados por N. elegantalis. As variáveis peso, pH e Brix $^{\circ}$ dos frutos não foram alteradas com o ensacamento de cachos com TNT.

\section{REFERÊNCIAS}

ABAURRE, Maria Elizabete Oliveira. Práticas culturais. In: Tomate. Vitória: Incaper, 2010. Cap. 6, p. 133-148.

BLACKMER, J. L.; EIRAS, A. E.; SOUZA C. L. M. de. Oviposition preference of Neoleucinodes elegantalis (Guenée) (Lepidoptera: Crambidae) and rates of parasitism by Trichogramma pretiosum Riley (hymenoptera: Trichogrammatidae) on Lycopersicon esculentum in São José de Ubá RJ, Brazil. Neotropical Entomology. Londrina, v. 30, n. 1, p. 89-95, 2001.

FAO - Food and Agriculture Organization of the United Nations. Countries by commodity, 2018. Disponível em http://www.fao.org/faostat/en/\#rankings/countries_by_commodity. Acesso em: 11 jul. 2019.

FILGUEIRAS, R. M. C.; PASTORI, P. L; PEREIRA, F. F.; COUTINHO, C. R.; KASSAB, S. O.; BEZERRA, L. C. Agronomical indicators and incidence of insect borers of tomato fruits protected with non-woven fabric bags. Ciência Rural. Santa Maria, v. 47, n. 6, p. 1-6, mar. 2017.

HACHMANN, T. L.; ECHER, M. M.; DALASTRA, G. M.; VASCONCELOS, E. S.;

GUIMARÃES, V. F. Cultivo do tomateiro sob diferentes espaçamentos entre plantas e diferentes níveis de desfolha das folhas basais. Bragantia. Campinas, v.73, n.4, p. 399-406, jul. 2014.

IBGE - Instituto Brasileiro de Geografia e Estatística. Tomate, 2018. Disponível em < https://sidra.ibge.gov.br/tabela/1618 >. Acesso em 11 jul. 2019.

JORDÃO, A. L.; NAKANO, O. Ensacamento de frutos de tomateiro visando ao controle de pragas e à redução de defensivos. Scientia Agricola. Piracicaba, v. 59, n. 2 p. 281-289, jun. 2002.

LEITE, G. L. D.; FIALHO, A.; ZANUNCIO, J. C.; JÚNIO REIS, R.; DA COSTA C. A. Bagging tomato fruits: A viable and insect damage in organic production. The Florida Entomologist.

Saint Augustine, v. 97, n. 1, p. 50-60, mar. 2014.

LIPP, J. P.; SECCHI, V. A. Ensacamento de frutos: uma antiga prática ecológica para controle da mosca-das-frutas. Agroecologia e Desenvolvimento Rural Sustentável. Porto Alegre, v.3, n.4, p. 53-58, dez. 2002.

MARCANO, R. Ciclo biológico del perforador del fruto del tomate Neoleucinodes elegantalis (Guenée) (Lepidoptera: Pyralidae), usando berenjena (Solanum melongena) como alimento.

Boletím de Entomología Venezolana. Caracas, v.6, n.2, p. 135-141, dez. 1991.

MONTILA, A. E. D.; SOLIS, M. A.; KONDO, T. The tomato fruit borer, Neoleucinodes elegantalis (Guenée) (Lepidoptera: Crambidae) an insect pest of Neotropical solanaceous fruits. 
In: PEÑA, Jorge E. (Org.). Potential invasive pests of agricultural crops. Wallingford: CABI, 2013. Cap. 8, p 137-159.

PICANÇO, M. C.; BACCI, L.; CRESPO, A. L. B.; MIRANDA, M. M. M.; MARTINS, Júlio C. Effect of integrated pest management practices on tomato production and conservation of natural enemies. Agricultural and Forest Entomology. Hoboken, v. 9, n. 4, p. 327-355, ago. 2007.

PRATISSOLI, D. Guia ilustrado de pragas da cultura do tomateiro. Alegre: Unicopy, 2015. $45 \mathrm{p}$.

WAMSER, A. F.; BECKER, W. F.; SANTOS, J P.; MUELLER, S. Influence of the training systems of tomato plants on the incidence of diseases and insect-pests. Horticultura Brasileira. Recife, v. 26, n.2, p. 180-185, jun. 2008. 